Abodovsky N. y cols.

Rev. Chil. Pediatr. 66 (3); 140-144, 1995

\title{
Detección precoz de hipotiroidismo congénito en Chile. Resultados en 24 meses
}

\author{
Nelly Abodorsky G. ${ }^{1,2}$; Ximena Vivanco W.'; Ximena Cuello A. \\ Pamela Pérez O.'; Gabriel Lobo S. ${ }^{3}$; Jorge Brantes M. ${ }^{4}$; Jorge Pacheco T. ${ }^{5}$ \\ Results on the first two years of the chilean neonatal screening \\ programme for congenital hypothyroidism 24 month (1992 - 1994)
}

The chilean programme for neonctal screening of corgenula hypotmyrodism wos stcred by march 1892 under the sponsor of the Narional Health Minis'ry. Results after two yeor applica:ion lill marc 1994 at the Metropolitan and its necrby Sixth geopolirical egions of the country are reponteo. All newbon infants were screened for thyord stimulating hormone (TSH) 'evels in blaod somples cbrained l'omi heel puncture, by an mmine radiomeric csay on filler paper sFo' metrod, berwieen 2 and 5 doys after birth. All suspecred ecses were recallec ?cr postneonatcl confimatory lests in blood by immune radionerric asay of TSH, rodioimmune asoy of TA and thyroid Te99 seintigrcphic scan. Cutcff points of TSH were stoblished of $\geq 20 \mathrm{mUl} / \mathrm{l}$ for egrly neonotal blood samoles in filler poper and $>10 \mathrm{mUl} / \mathrm{i}$ for Dostrecnata! blood samples. Along tre two year ceriod of opplicction, 130383 necnates were screened 158.9 and $988 \%$ respectively $5^{2}$ the whole recorded births in the first anc second vearl and $000^{\circ}$ them $(0.05 \%)$ were recalled:

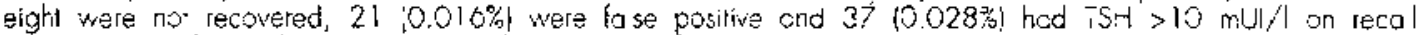
examiration. Or these lale. 23 ware confirmed cases of congen tal hypothycidis.n and 14 were considered to have hiperthyrotropinemia (thiperTSH\}. Along follow up. 8/14 parienls with hyper TSH we e cherwards clasified as compensated congenik' hypothyrodism with abnormal thyroid scintigraphy, three wee coses of trensienl TS 4 exess and the last three remained suspects of conge-iral hypothyroidism. A total of 31 cases of congenital hypothyroidism or

4200 newsom infants were thus identfied, with c female to male ratio of 25 to 6 . As thyrsid scintigraphy three patiente sthowed absenl thyroid lissue [10.34\%), one $13.4 \%$, had minimal Tcog uplake, 10 had eccpic thyroid glands (55.17\%) seven had gciter [24. ${ }^{14 \%}$ ) and two $0.9 \%$ had nornol scinigraph c scons. Averoge age ar slort of L-thyoxine "epiacement therapy; dosage $101015 \cdot 9 \cdot \mathrm{kg}$ pe dayh was 19.74 doys. Psycromoto" deve opment as measured by Bayley sca e test anc nesroiog cal examination has peen normal, excep in one case whose reclment begun late, a ace 73 coys.

(Key words: congeritcl rypothyroidssm, neonatal screening.!

El hipotiroidismo congénito ( $\mathrm{HC}$ ) es la causa más común de retardo mental prevenible. Ocure en aproximadamente 1 de 3500 a 4000 recién nacidos $\left(\mathrm{RN}^{\mathrm{T}}\right)$ vivos. Su etiología incluye disgenesias tiroideas (aplasia, hipoplasia, ectopia), dishormonogenesis tiroideas, deficiencia hipotálamo hipofisiaria, hipotiroidismos transi-

1. Servicio de Pediatria, Departamento de Endocrinología Infantil, Hospital San Juan de Dios.

2. Departamento de Pediatría. División Occidentc, Faculand de Medicipa, Unjersidad de Chile.

3. Laboratorio de Medicina Nuclear. Hospital San Juan de Dios.

4. Coordinador Programa Servicio Salud Occidente.

5. Tecnólogo Médico. Departamento de Medicina Nuclear, Hospital San Juan de Dios. torios, déficit de TBG y alteraciones de los receptores celulares ${ }^{1.2}$.

Las hormonas tiroideas son esenciales para el desarrollo y crecimiento del cerebro. La maduración del sistema nervioso central termina alrededor de los dos primeros años de vida y aproximadamente la mitad de ella se alcanza en los primeros seis meses. Por lo tanto el diagnóstico y el tratamiento precoz (antes de la edad de un mes) del hipotiroidismo congénito son muy inportantes para prevenir el déficit intelectual que deriva del defecto ${ }^{3}$. El diagnóstico clíniço de hipotiroidismo en el recién nacido es muy difícil; los signos precoces son generalmente inespecíficos o velados. Por esta razón el rastreo masivo de hipotiroidismo en neonatos se realiza me- 
diante mediciones, más sensibles y específicas, de hormonas tiroideas o hipofisiarias con micrométodos.

Los coeficientes intelectuales de los pacientes cuyo tratamiento se inicia antes de la edad de treinta días son normales ${ }^{3.4}$. En efecto, en niños que recibían tratamiento temprano (promedio 24 días de vida) el dicho coeficiente era. a los seis años, $109 \pm 13$ ante $110 \pm 13$ en sujetos normales, $y$ entre los nueve y diez años de edad, $106 \pm 12,2$ ante $109 \pm 13,5^{5}$.

El rastreo neonatal de hipotiroidismo se hace en forma rutinaria en Estados Unidos de América y Canadá, en la mayoría de los países de Europa del Oeste, Escandinavia, Japón, Israel, Australia y Nueva Zelandia y 10 a 12 millones de niños por año se examinana en todo el mundo para detectar la alección como una forma de prevenir retardo mental ${ }^{6}$.

En marzo de 1992 se inició en la Región Metropolitana y en su vecina sur, la VI Región de Chile, un programa de rastreo de la enfermedad, de acuerdo con las recomendaciones de la Organización Mundial de la Salud (OMS) ${ }^{\dagger}$. como primera etapa de un plan nacional financiado por el Ministerio de Salud. En esta comunicación se da cuenta de los resultados obtenidos en dos años de observación (marzo 19921994).

\section{Material y Método}

Entre el 1 de marzo de 1992 y el 28 de febrero de 1994 se analizaron $130 \quad 383$ muestras obtenidas de un total dc 196477 recièn nacidos registrados en la Región Metropolitana y VI Región del pars. El rastreo se hizo mediante determinación de TSH por ensayo radioinmunométrico (lRMA) en una gota de sangre obtenida por punción de talón, a las 48 y 72 horas de vida en los nacidos de térmito y al quinto día en los prematuros. La sangre extraida se depositó en una tarjeta de papel filtro Macherey 818 , diseñada para este propósito y se dejó secar a lemperatura aunbiente. Las concentraciones de TSH se unidieron en muestras de $6 \mathrm{Jnm}$ de diámetro utilizando un método inmunoradiométrico de doble sitio o localización, en fase sólida, empleando reaclivos comerciales CIS Internacional ${ }^{8}$. To. dos los recién nacidos cuya concentración sanguínea de TSH fue igual o superior a $20 \mathrm{mb}$ Hl (punco de corte) fueron vueltos a llamar para efectuar confirnación en suero con TSH por IRMA y T4 por radioinmunoensayo (RIA), empleando juegos comerciales T4. Total Baxter USA para T4 y Amersham, England, para TSH. En consideracion a la edad de los niños vueltos a llamar al momento de la confirmación, se estableció un nuevo punto de corte para TSH en suero de $10 \mathrm{~m} U \mathrm{l} / \mathrm{y} \geq 10 \mu \mathrm{g} / \mathrm{dl}$ como concentración nor- mal de T4. Junto con el examen de confímación se realizo cintigrafía tíroidea con tecnecio 99 (Tc99) y se evaluó cdad ósea con radiografía en rodilla. En los casos tatalogados como hipotiroidismo congénito (TSH $>10 \mathrm{mUL} / \mathrm{l}$ y $\mathrm{T} 4<10$ $\mu \mathrm{g} / \mathrm{dl}$ o cintigrafía tiroidea anormal, aun con $\mathrm{T} 4$ normal) según los resultados de los exámenes de confirmación, se injció inmediatamente tratamiento con levotiroxina en dosis inicial de 10 a $15 \mu \mathrm{g} \cdot \mathrm{kg}$ - dít. disminuyéndola gra dualmente hasta 5 o $6 \mu \mathrm{gg} \cdot \mathrm{kg}$ - día a los 12 meses de vida. A lodos los pacientes se les aplicó un protocolo de seguimiento que incluía evolución clínica; antropometria, interpretada de acuerdo a las tablas de ta OMS para peso, talta y circunferencia craneana; concentraciones scricas de TSH por IRMA $y$ T4 por RIA: edad ósea con radiografía de carpo según atlas de Greulich y Py̧le: desarrollo neurológico mediante examen físico realizado por un neurologo pediátrico y psicometría mediante pruebas de Baylcy $y$ Stanford Binet, aplicadas por un psicólogo.

\section{Resultados}

Se practicaron 130383 exámenes de TSH en papel filtro. La cobertura promedio el primer año fue $58,9 \%$ y el segundo año de $98,8 \%$. Se encontró TSH > $20 \mathrm{mULI}$ en papel filtro en 66 RN $(0,05 \%)$, que fueron rellamados; 8 no se presentaron. En los demás se realizó confirmación en suero con T4 y TSH, resultando, definitivamente, normales (falsos positivos) las concentraciones séricas de 21 casos $(0,016 \%)$. En 37 recién nacidos $(0,028 \%)$ TSH fue $>10 \mathrm{mUV} / \mathrm{t}$ en el examen de confirmación, en 23 de ellos la concentración sérica de $\mathrm{T} 4$ era $<10 \mu \mathrm{g} / \mathrm{dl}$, por lo que se catalogaron de inmediato como hipotiroidismo congénito. En los 14 restantes la concentración de T4 era normal y ellos fueron consignados como hipertirotropinemias.

La concentración sérica de TSH en papel filtro fue $29.92 \pm 9$ (márgenes 20,2 a 48,2 ) $\mathrm{mUl} / /$ para los falsos positivos, $109,24 \pm 53,7$ (márgenes 26,7 a 521,4$) \mathrm{mUL} / \mathrm{l}$ en los hipotirodismos congénitos confirmados y $31,54 \pm 23,4$ (márgenes 20,9 a 82,2 ) mUL/ en los calificados de hipertirotropinemia.

La cintigrafía tiroidea estaba alterada en los 23 casos con diagnóstico inicial de hipotiroidismo congénito y correspondía a ectopia en 16 $(55,1 \%)$ pacientes; bocio en $7(24,14 \%)$ niños; agenesia en $3(10,34 \%)$ recién nacidos; disminución de concentración de TC99 en un $(3,4 \%)$ caso y normal en $2(6,9 \%)$. En seis de los 14 casos calificados como hipertirotropinemia la cintigrafía dio resultados anormales (3 ectopias, 2 bocios y 1 ausencia de concentración del ra- 
diofármaco), todos ellos se consideraron hipotiroidismos congénitos compensados y recibieron tratamiento. En tres de esos 14 pacientes la concentración de Tc99 estaba disminuida (cintigrafía no concluyente o dudosa), en dos la concentración de TSH sé normalizó a los 5 y 10 meses de vida, clasificándoseles como hipertirotropinemias transitorias, y el otro niño se perdió del control. En dos de los mismos 14 casos con hipertirotropinemia cuya cintigrafía era normal, el T4 descendió a los 2 meses de edad, por lo que se diagnosticó hipotiroidismo congénito de comienzo tardío y se indico tratamiento. La cintigratía tiroidea no se realizó en tres casos de hipertirotropinemia, en uno se normalizó la concentración sérica de TSH (hipertirotropinemia Iransitoria), otro está en tratamiento por persistir con TSH elevado y el tercero se perdió de control. En resumen, un total de 31 pacientes con hipotiroidismo congénito, 25 de sexo femenino, configurando una incidencia de $1 / 4206$ reciés nacidos vivos.

La edad 6 sea medida en la radiografía de rodilla fuc normal en $18(85.7 \%)$ y atrasada en 3 $(14,3 \%)$ casos de hipotiroidismo congénito en que se realizó.

El tratamiento con levotiroxina se inició, con una sola excepcion, antes de los 30 días de edad (promedio 19,74, márgenes 5 a 73 días). Sólo un caso se trató a los 73 días. El tratamiento fue vigilado con determinaciones de T4 y TSH en el suero, que alcanzaron concentraciones normales a los 22 días (promedio) después de iniciado el tratamiento. La concentración máxima promedio de T4 alcanzada en este período fue 16,3 $\mu \mathrm{g} / \mathrm{dl}$, márgenes 10 a $25 \mu \mathrm{g} / \mathrm{dl}$, mediana 15,95 $\mu \mathrm{g} / \mathrm{d} 1$, agrupándose el mayor número de casos entre 14 y $18 \mu \mathrm{g} / \mathrm{dl}$.

EI examen neurológico realizado por especialista fue normal en todos los casos, excepto aquel en que el tratamiento fue iniciado tardíamente. La psicometría mediante prueba de Bayley se realizố a 20 niños con hipotiroidismo congenito a una edad de 10,2 meses (media). márgenes 2 y 18 meses, mediana $1 \nmid$ meses. El promedio del coeficiente de desartollo mental obtenido fue 102.5 , márgenes 80 y 117 (normal > 84) 9; cl promedio del coeficiente de desarrollo motor fue de 98,1 , márgenes 72 y 136) (normal $>84)^{9}$. Sólo en el paciente que se trató tardíamente. los cuocientes de desarrollo estaban bajo el mínimo normal.

\section{Comentario}

La buena cobertura es fundamental para el funcionamiento de un programa de esta naturaleza. La baja cobertura registrada en la serie expuesta durante el primer año de aplicación se debíb a la incorporación paulatina al programa de las diferentes áereas de la Región Metropolitana y VI Región, pero al segundo año fue satisfactoria.

La obtención de las muestras al tercer día de vida en los nacidos de término y el quinto día en los pretérmino se justifica en el aumento fisiologico de TSH que ocurre en las primeras horas de vida. La frecuencia de $0,05 \%$ de rellamados y los puntos de corte elegidos para la concentración sérica de TSH coinciden con los establecidos en la mayoría de los programas de detección precoz de hipotiroidismo congénito ${ }^{1.6}, 9,10$. Los márgenes de normalidad para el $\mathrm{T} 4$ de confirmación son, sin embargo, diferentes en distinlos grupos de trabajo. En este caso se eligió la concentracion de $\geq 10 \mu \mathrm{g} / \mathrm{dl}$, considerando que para $97 \%$ de los hipotiroideos el límite superior de T4 es $10 \mu g / d^{16}$.

La proporción de casos confirmados de hipotiroidismo congénito enfatiza la importancia de considerar a todo recién nacido con TSH elevado y T4 normal como potencialmente hipotiroideo. Por esta razón la cintigrafía tiroidea debe realizarse simultáneamente con los exámenes de comprobación, pues ella y el seguimiento clínico permitirán detectar a los hipotiroidismos congénitos, cuya incidencia fuc similar a la descrita en otros informes de rastreo ${ }^{1 !}$.

La etiologia del hipotiroidismo congénito fue también semejante en esta serie que en otras precedentes, prevaleciendo las disgenesias. Llama la atención el alto porcentaje de bocio, cuya causa precisa requiere más estudio. Las concentraciones más bajas de $\mathrm{T} 4$, detectadas en los caso de agenesia tiroidea, otorgan especial importancia al tratamiento precoz, dado el mayor riesgo de dario neurológico que el trastorno conlleva.

Para que el desarrollo neurológico y psicomotor sean normales en los pacientes con hipotiroidismo congénito se requiere iniciar su tratamiento alrededor de los 15 días de vida con dosis de T4 entre 10 y $15 \mu \mathrm{g} / \mathrm{kg}$, alcanzando concentraciones séricas de 10 a $16 \mu / \mathrm{dl}$ al término de una o dos semanas ${ }^{6,12-15}$. En los casos que se 
comentan, la normalización de $\mathrm{T} 4$ y la frenación de TSH se registraron en aproximadamente tres semanas después de iniciar el tratamiento. 10 que probablemente guarda relación con los buenos resultados obtenidos en las pruebas de desarrollo psicomotriz. El T4 sérico en algunos casos alcanzó concentraciones sobre el límite superior normal de $16 \mu \mathrm{g} / \mathrm{dl}$, pero la vigilancia periódica permitió ajustar las dosis oportunamente, sin que ningún niño presentase signos de hipertiroidismo, adelanto de la edad ósea o craneosinostosis, corroborando así la necesidad de los controles frecuentes.

En las hipertirotropinemias aisladas es importante establecer si son transitorias o expresión de hipotiroidismo compensado. La forma transitoria ha sido recientemente descrita, su incidencia sería $1 / 18000$ recién nacidos y su etiopatogenia no es bien conocida ${ }^{16-19}$. En nuestra serie la incidencia de hipertirotropinemia transitoria fue más baja. Los demás casos se consideraron sospechosos de hipotiroidismo congénito, sea porque su cintigrafía tiroidea dio resultados dudosos o porque no fue posible hacerla, realizándoseles tratamiento. Estos últimos pacientes así tratados deberán ser reevaluados a los tres años de edad, para establecer el diagnóstico definitivo, de acuerdo a las recomendaciones de la Academia Americana de Pediatría ${ }^{12}$

El desarrollo neurológico e intelectual adecuado en nuestros casos demuestra la importancia del tratamiento precoz, antes del primer mes de vida. La incidencia del hipotiroidismo congénito aquí descrita, justifica la aplicación del programa en todo el país. No obstante, la vigilancia clínica permanente mantiene su validez, ya que cierta proporción de los hipotiroidismos congénitos no es delectada por el procedimiento de rastreo.

\section{Resumen}

Se informa el resultado de dos años de aplicación en las Regiones Metropolitana y VI de Chile, de un plan de rastreo masivo de hipotiroidismo congénito en recién nacidos, financiado por el Ministerio de Salud. Se midió TSH por ensayo radioinmunométrico en sangre obtenida de punción del talón y recolectada en papel filtro, de 130383 recién nacidos entre los 2 y 5 días de vida, con coberturas de 58,9 en el primero y $98,8 \%$ en el segundo año de aplicación del programa. Se rellamaron los 66 niños cuya concentración sérica de TSH fue igual o mayor a $20 \mathrm{mUI} / \mathrm{l}$ para realizar examen de confirmación en suero con TSH por IRMA y T4 por radioinmunoensayo y simultáneamente cintigrafía tiroidea con Tc99. Ocho niños rellamados no respondieron, $21(0,016 \%)$ resultaron falsos positivos y $37(0,028 \%)$ tenían TSH $>10 \mathrm{mUL} /$ en el examen de comprobación. De estos últimos, 23 tenían hipotiroidismo congénito y 14 hipertirotropinemia. En el seguimiento de los casos de hipertirotropinemia se pesquisaron otros ocho de hipotiroidismo congénito compensado, tres de hipertirotropinemia transitoria $y$ tres continuaron siendo sospechosos de hipotiroidismo congénito. En total se pesquisaron 31 casos de hipotiroidismo congénito, incidencia de 1/4 206 RN vivos, proporción de 25:6 entre mujeres y varones. La cintigrafía tiroidea mostró agenesia en tres pacientes $(10,34 \%)$, ectopia en $16(55,17 \%)$, bocio en siete $(24,14 \%)$, en uno babía sólo disminución de concentración de Tc99 (3.4\%) y en dos no existían alteraciones cintilográficas $(6,9 \%)$. El tratamiento con levotiroxina 10 a $15 \mu \mathrm{gg} \cdot \mathrm{kg}$ se inició a la edad de 19,74 días. promedio. Sólo un caso se trató después de un mes, a los 73 días de edad. La psicometría (prueba de Bayley y Stanford Binet) y el examen neurológico fueron normales en todos los casos, excepto en el caso que se trató tardíamente.

(Palabras clave: hipotiroidismo congénito, rastreo neonatal.)

\section{Referencias}

1. Fischer $D$ : Screening for congenital hypothyroidism. Hosp Prace 1977; 73-78.

2. Barnes $N D$ : Screcning for congenital hypothyroidism the first decade. Arch Dis Child 1985; 60: 587-592

3. Wh R. Lurgt RH, Qin $Q$. Torresuni $T$, Rochiccioli $P$, and Larson A: Mental development in congenutal hyporhyroidism after neonatal screening. Arch Dis Child 1987; 62: 1050-1055.

4. Glorieux $J 1$ Dussautt $J M$, et al.: Follow-up at ages 5 and 7 years on ineatal development in children with hypothyroidism detected by Quebec Screening Progran. J Pediatr 1985: 107: 913-918.

5. Arnold $M B$ er al.: New England Congenital Hypothyroidism Collaborative. Elementary school performance of children with congenital hypothyroidism. J Pediatr 1990; 116: 27,-32. 
6. Fischer D: Management of, Congenital Hypothyroidism. J Clin e Endocr Metab 1991: 72: 523-\$29.

7. Hollamon $N$ : Newbom screening for inbom errors of inetabolism. Pediatr Clin North 1978; 25: 411-420.

8. Hernández V. Pacheco J, Vivanco $X$, Cuello $X, A b o$ dovisky $N$, Lobo $G$ : Valores de TSH neonatal en una población rural. Bol Hosp S J de Dios 1993; 40 (2): 69-72.

9. Bayley N: Bayley scales of infants development. Second edition 1993. Psichological Cotporation. San Antonio, USA.

10. Zitung YQ: Experience in neonatal screening for congenital hipothyroidism. Chin Med J (Engl) 1993; 106: 216-219

11. Fisher $D$ : Effectiveness of newborn screening program for congenital hypothyroidism: prevalence of missed cases. Pedial Clin North Am 1987: 34: 881889.

12. American Academy of Pediatrics. Newborn Screening for Congenilal Hypothyroidism: Recommended Guidelines. Pediatrics 1993; 91: 1203-1209.

13. Pharoah POD, Medden MP: Audit of screening for congenital hypothyroidism. Arch Dis Child 1992; 67: 1073-1076.

14. Germak JA, Foley Jr. TP: Longitudinal assessment of L-thyroxine therapy for congenital hypothyroidism. J Pediatr 1990; 117: 211+219.

15. Heyerdati S. Bengt Frode K, Sverre O: Intelectual development in children with congenital hypothyroidism in telation to recomended thyroxine treatment. J Pediatr 1991: 118: 850-857.

16. Alenzadeh $R$. Friedman S, Forn P. Recker B, Liffititz $F$ : Is there compensated hypothyroidism in infancy? Pediatrics 1992; 90: 207-211.

17. Miki $K$ : Transient infantile hipentirotropinemia. Arch Dis Child 1989; 64: 1177-J182.

18. Miyti $K$ : Hiperthyrotropinemia with normal thyroid hormone concentration in newbom babies, In Nanise H. Iric M. eds, Neonatal Screening Amsterdam, the Netherlands: Excerpla Medica: 1983: 44-49. Excerpta Medica International Congress Series 606.

19. Tyfiefd LA: Persistente hyperticotropinemia since the neonatal period in clinically euthyroid children. Eur I Pediatr 1991; 150: 308-309.

\section{AVISO A LOS AUTORES}

Con el objeto de dar prioridad a los trabajos de investigación, en vista de las limitaciones de espacio de la Revista Chilena de Pediatría, el Comité Editorial ha acordado restringir la impresión de casos clínicos a un máximo de dos por cada número. 